\title{
Identification of important genes and pathways leading to poor prognosis of non-small cell lung cancer using Integrated Bioinformatics
}

\section{Shengjin Cui}

Shenzhen Hospital,Southern Medical University

\section{Feixia Huang}

University of Hong Kong-Shenzhen Hospital

\section{Fan Yu}

Shenzhen Hospital,Southern Medical University

\section{Shihui Jian}

Guangzhou Huayin Medical Inspection Center Co.Ltd.

\section{Lijun Zhang}

Shenzhen Hospital,Southern Medical University

\section{Xintong Liu}

Shenzhen Hospital,Southern Medical University

\section{Siping Chen}

Shenzhen Hospital,Southern Medical University

Yiwen Zhou ( $\nabla$ yiwenzhou21@aliyun.com )

Shenzhen Hospital,Southern Medical University

\section{Lijia Xiao}

Shenzhen Hospital,Southern Medical University

\section{Keywords:}

Posted Date: June 24th, 2020

DOl: https://doi.org/10.21203/rs.3.rs-34440/v1

License: (c) (i) This work is licensed under a Creative Commons Attribution 4.0 International License. Read Full License 


\section{Abstract}

The authors have requested that this preprint be removed from Research Square. 